\title{
Benign Prostatic Hyperplasia and Male Lower Urinary Tract Symptoms: Epidemiology and Risk Factors
}

\author{
J. Kellogg Parsons
}

Published online: 7 September 2010

(C) The Author(s) 2010. This article is published with open access at Springerlink.com

\begin{abstract}
The epidemiology of benign prostatic hyperplasia (BPH) and male lower urinary tract symptoms (LUTS) has evolved considerably during the past several years. The term LUTS describes a distinct phenotype and allows for a broad epidemiologic description of urinary symptoms at a population level. Although it is becoming the preferred term for studying urinary symptoms in populations, LUTS remains interconnected with $\mathrm{BPH}$ in the literature. The incidence and prevalence of BPH and LUTS are increasing rapidly as the US population ages. BPH and LUTS are associated with serious medical morbidities, an increased risk of falls, depression, diminished health-related quality of life, and billions of dollars in annual health care costs. Although age and genetics play important roles in the etiology of BPH and LUTS, recent insights at the population level have revealed that modifiable risk factors are likely key components as well. Serum dihydrotestosterone, obesity, elevated fasting glucose, diabetes, fat and red meat intake, and inflammation increase the risk; vegetables, regular alcohol consumption, exercise, and NSAIDs decrease the risk.
\end{abstract}

Keywords Benign prostatic hyperplasia .

Lower urinary tract symptoms .

American Urological Association Symptom Index · BPH .

LUTS · Epidemiology · Risk factor · Exercise ·

Physical activity $\cdot$ Metabolic syndrome $\cdot$ Modifiable $\cdot$

Obesity $\cdot$ Diabetes

\footnotetext{
J. K. Parsons $(\square)$

In care of Leslie Parker, Division of Urology,

University of California, San Diego,

200 West Arbor Drive \#8897,

San Diego, CA 92103-8897, USA

e-mail: leparker@ucsd.edu
}

\section{Introduction}

The epidemiology of benign prostatic hyperplasia (BPH) and male lower urinary tract symptoms (LUTS) has evolved considerably during the past several years. The incidence and prevalence of BPH and LUTS are increasing rapidly as the US population ages. BPH and LUTS are associated with serious medical morbidities, an increased risk of falls, depression, diminished health-related quality of life, and billions of dollars in annual health care costs. Although age and genetics play important roles in the etiology of BPH and LUTS, recent insights at the population level have revealed novel risk factors that present new opportunities for treatment and prevention. Indeed, an exciting notion is that to a large extent, BPH and LUTS pathogenesis may be driven by modifiable risk factors, including serum dihydrotestosterone (DHT), obesity, glucose homeostasis, diet, exercise, and inflammation.

\section{Identification and Definition in Epidemiologic Studies}

LUTS represent a cluster of chronic urinary disorders that occurs among $15 \%$ to $60 \%$ of men older than 40 years of age [1-4]. Specific symptoms associated with the LUTS complex include frequency, urgency, nocturia, difficulty initiating urination, sense of incomplete bladder emptying, decreased force of stream, and interruption of stream.

The most common etiology of male LUTS is BPH. A persistent conundrum in designing and interpreting population-based studies of urinary symptoms in older men is case definition. In the past, chronic urinary symptoms in older men were uniformly ascribed to-and defined as - BPH. There are at least two reasons why BPH is a problematic outcome measure for population studies. First, BPH has many different definitions, including 
histologic analysis of prostate tissue, radiographically determined prostate enlargement, decreased urinary flow rates, history of noncancer surgery of the prostate, physician-diagnosed BPH, and urinary symptoms. Heterogeneity in defining BPH makes validation across study populations difficult. Second, BPH excludes other potential causes of urinary symptoms in older men, such as interstitial cystitis and detrusor instability.

LUTS describes a distinct phenotype of a group of disorders affecting the prostate and bladder that share a common clinical manifestation. The term LUTS allows for a broad epidemiologic description of urinary symptoms at a population level without identification of organ- or diseasespecific etiologies. This approach allows consideration of LUTS within a macroscopic context using a uniform definition unbiased by variable definitions of BPH and other diseases. In recent observational studies, LUTS has become the preferred term for studying urinary symptoms in populations [2-4]. Still, as most men with LUTS have BPH and most men with BPH have LUTS, the two terms remain inextricably interconnected in the contemporary treatment and study of urinary disorders in older men. Therefore, this review discusses BPH and LUTS as separate outcomes describing the same spectrum of male urinary disorders.

The most commonly used measures of LUTS in epidemiologic studies, and the primary instruments by which $\mathrm{BPH}$ is diagnosed in clinical practice are the American Urological Association Symptom Index (AUASI) and its internationally validated counterpart, the International Prostate Symptom Score (I-PSS). The AUASI and I-PSS are robust and reliable quantitative instruments for measuring urinary symptoms in men. As part of its clinical guidelines, the American Urological Association recommends the routine use of the AUA-SI or I-PSS in the clinical evaluation of patients with suspected BPH (http:// www.auanet.org/guidelines).

\section{Epidemiology of Lower Urinary Tract Symptoms and Benign Prostatic Hyperplasia}

\section{Incidence and Prevalence in an Aging Population}

The prevalence of BPH and LUTS rises markedly with increased age. BPH affects $70 \%$ of US men $60-69$ years of age and $80 \%$ of those 70 years of age or older [1]. In the Boston Area Community Health survey, LUTS prevalence increased from $8 \%$ in men 30-39 years of age to $35 \%$ in men $60-69$ years [2]. In the Rancho Bernardo study, $56 \%$ of men $50-79$ years of age, $70 \%$ of men $80-89$ years of age, and $90 \%$ of men 90 years of age or older reported LUTS [4]. Other population-based studies have demonstrated similar trends [3].
LUTS incidence among older men is also considerable. In the Osteoporotic Fractures in Men cohort, a prospective study of 6,000 community-dwelling men older than 65 years of age, $29 \%$ of those without LUTS at baseline developed clinically significant LUTS within 2 years of follow-up; among those 80 years of age or older, this proportion increased to $34 \%$ [5]. In the Olmsted County cohort, $14 \%$ of men without LUTS at baseline subsequently reported moderate or severe symptoms within 18 months of followup, and $22 \%$ reported moderate or severe symptoms within 42 months of follow-up [6]. Similarly, $21 \%$ of Japanese, $26 \%$ of black American, and $20 \%$ of Austrian men with no or mild LUTS at baseline reported worsened symptoms after 3, 4, and 5 years of follow-up, respectively [7-9]

The prevalence and incidence of BPH and LUTS in the United States increased steadily between 1994 and 2000 (http://www.UDAonline.net). Between 1998 and 2007, the age-adjusted prevalence of BPH among hospitalized patients in the United States nearly doubled (Stroup and Parsons, unpublished data). Increases in BPH and LUTS prevalence and incidence are occurring within the context of an aging population. By 2030, 20\% of the US population will be 65 years of age or older, a figure that will include more than 20 million men. Significantly, the fastestgrowing segment of the older adult population is the oldest age group: those older than 85 years of age. Current estimates indicate that the number of individuals 80 years of age or older in the United States will rise from 9.3 million in 2000 to 19.5 million in 2030, an increase of more than $100 \%$ [10]. These trends portend substantial increases in the number of incident and prevalent LUTS and BPH cases within the next few decades.

\section{Public Health Impact of Lower Urinary Tract Symptoms} and Benign Prostatic Hyperplasia

It is important to consider the substantial adverse consequences of LUTS and BPH for the global health of older men. Although it may be tempting to dismiss LUTS and BPH as relatively harmless disorders representing more of an inexorable, if inconvenient, effect of aging, this perception belies the substantial medical, psychological, and economic burdens of these conditions. Despite widespread use of medical therapy (http://www. UDAonline.net), BPH remains associated with a substantial prevalence of urinary infections, bladder stones, urinary retention, and acute renal failure (Stroup and Parsons, unpublished data). In addition, the adverse health effects of LUTS and BPH encompass serious nonurologic conditions. Men with severe LUTS have a $63 \%$ increased risk of falling at least twice within 1 year compared with men with no symptoms [11]. Falls in older adults are associated with debilitating morbidities, including pain and fractures. LUTS 
is also associated with a significantly diminished quality of life, depression, and impairment in instrumental activities of daily living $[3,12]$.

Another important public health issue is the cost associated with diagnosis and treatment. In 2000, the most recent year for which comprehensive data are available, $\mathrm{BPH}$ generated 1.1 billion dollars in direct health care expenditures and accounted for more than 4.4 million office visits, 117,000 emergency department visits, 105,000 hospitalizations, and 21 to 38 million hours in lost productivity in the United States. During the same year, the direct costs of treatment for overactive bladder in men totaled 1.8 billion dollars, with another 3.9 billion dollars directed to ancillary health care expenditures, including treatment for urinary tract infections. Estimated annual costs of BPH treatment currently total 3.9 billion dollars $[1$, $13,14]$.

The high prevalence of LUTS in the older male population, its detrimental impact on male health, and the substantial costs associated with diagnosis and treatment underscore the significance of this problem, particularly in an era of rapidly increasing male longevity.

\section{Risk Factors Associated with Lower Urinary Tract Symptoms and Benign Prostatic Hyperplasia}

On a population level, there are five broad categories of risk factors for BPH and LUTS (Fig. 1). Aside from age, the other categories are genetics, sex steroid hormones, modifiable lifestyle factors, and inflammation.

\section{Genetics}

Evidence suggests that there are strong genetic components to both BPH and LUTS. One case-control analysis, in which participants were men younger than 64 years of age who underwent surgery for BPH, noted fourfold and sixfold increased age-specific risks of BPH surgery among all male relatives and brothers, respectively, of cases. These inves-

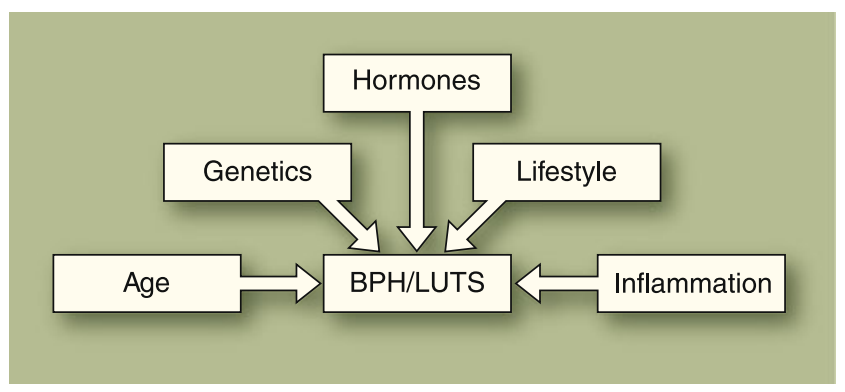

Fig. 1 Broad categories of epidemiologic risk factors for benign prostatic hyperplasia (BPH) and lower urinary tract symptoms (LUTS) tigators further estimated that $50 \%$ of men undergoing surgery for BPH who were younger than 60 years of age had a heritable form of disease [15]. These findings and those of others have suggested an autosomal dominant pattern of inheritance [16]. Men with inherited forms of $\mathrm{BPH}$ tend to have larger prostates and younger age at onset than men with sporadic BPH [17].

Monozygotic twin concordance rates of $63 \%$ and $26 \%$ have been observed for LUTS and BPH, respectively, with one study estimating that genetic factors may contribute as much as $72 \%$ to the risk of high moderate or severe LUTS among older men $[18,19]$.

\section{Sex Steroid Hormones}

Prostate tissue is composed of two basic elements: a glandular element composed of secretory ducts and acini, and a stromal element composed primarily of collagen and smooth muscle. In BPH, unregulated cellular proliferation leads to increased prostate volume and increased stromal smooth muscle tone; increased prostate volume and stromal smooth muscle tone in turn cause physical compression of the urethra and mechanical obstruction of the bladder outlet. In prostatic secretory cells, the hormone 5- $\alpha$ reductase converts testosterone to DHT, a potent stimulator of prostate growth that, in addition to being necessary for prostate development, appears to play a central role in BPH pathogenesis. Multiple studies have explored associations of endogenous sex steroid hormones with BPH and LUTS.

\section{Testosterone}

At least seven observational studies have reported no associations and five inverse associations of serum testosterone (total, bioavailable, or free) with BPH or LUTS [20, $21 \cdot, 22]$. No studies to date have reported an increased risk of BPH or LUTS with higher serum testosterone levels. A prominent but theoretical concern of testosterone replacement therapy is the potential for it to exacerbate $\mathrm{BPH}$ and LUTS [23]. In this sense, these observations imply that higher serum testosterone concentrations do not promote $\mathrm{BPH}$.

\section{Dihydrotestosterone}

Several studies have noted an increased risk of BPH with increased serum concentrations of DHT and its metabolites. In one recent prospective study of community-dwelling men, those with the highest midlife levels of DHT had nearly three times the risk of subsequent BPH compared with those with the lowest levels [21•].

These results are consistent with those of three prior studies of serum concentrations of two DHT metabolites: 
17b-diol-glucuronide and androstanediol glucuronide. These metabolites are surrogate markers for DHT activity, with higher concentrations indicating increased and lower concentrations indicating decreased levels of DHT. Two cross-sectional studies and one prospective study have shown direct associations of these DHT metabolites with BPH or LUTS $[21 \bullet, 22]$.

The 5- $\alpha$ reductase inhibitors (finasteride and dutasteride) decrease serum concentrations of DHT [24] and prevent progression of clinical BPH [25]. However, it is not known whether finasteride or dutasteride will prevent incident $\mathrm{BPH}$.

\section{Estrogen}

No clear patterns of estrogen, LUTS, and BPH have yet emerged. Prior studies have reported positive, negative, and null associations of endogenous estrogens with $\mathrm{BPH}$ and LUTS $[21 \bullet]$.

\section{Other Hormones}

Other sex steroid hormones also have not demonstrated consistent links with LUTS or BPH. One study observed a positive association of DHEAS (dehydroepiandrosterone sulfate, a primary adrenal androgen with little intrinsic androgenic activity) with $\mathrm{BPH}$ and two inverse associations with LUTS [21•].

\section{Lifestyle}

A notable development in the epidemiology of BPH and LUTS is the recognition that modifiable lifestyle factors substantially influence the natural history of these conditions. Accumulating data intimate that many of the same metabolic disturbances associated with cardiovascular disease - and the lifestyle factors that modulate these disturbances-influence the risk of BPH and LUTS. These observations are important because they suggest the existence of modifiable pathways for BPH and LUTS that may present novel targets for prevention and treatment.

\section{The Metabolic Syndrome and Cardiovascular Disease}

The metabolic syndrome is a clinical constellation of metabolic abnormalities-obesity, glucose intolerance, dyslipidemia, and hypertension - that increases the risk of cardiovascular disease and results primarily from dietary and other lifestyle practices endemic to Westernized societies [26].

In one cohort, men diagnosed with at least three components of the metabolic syndrome had an $80 \%$ increased prevalence of LUTS compared with those with no components. Other studies have shown that men with heart disease are at significantly increased risk of clinical BPH and LUTS [27-29].

\section{Obesity}

Prior studies have consistently observed that increased adiposity is positively associated with prostate volumethat is, the greater the amount of adiposity, the greater the prostate volume. Body weight, body mass index (BMI), and waist circumference all have been positively associated with prostate volume in multiple different study populations [30-32]. In the Baltimore Longitudinal Study of Aging cohort, for example, each $1-\mathrm{kg} / \mathrm{m}^{2}$ increase in BMI corresponded to a $0.41-\mathrm{mL}$ increase in prostate volume. Moreover, obese $\left(\mathrm{BMI} \geq 35 \mathrm{~kg} / \mathrm{m}^{2}\right)$ participants had a 3.5 -fold increased risk of prostate enlargement compared with nonobese (BMI $<25 \mathrm{~kg} / \mathrm{m}^{2}$ ) participants [30]. A preponderance of published epidemiologic evidence also demonstrates that obesity increases the risks of BPH surgery, urinary symptom progression, initiation of BPH medical therapy, and LUTS [31-33].

\section{Diabetes and Disruptions in Glucose Homeostasis}

Disruptions in glucose homeostasis at multiple different levels-from alterations in serum insulin growth factor concentrations to diagnosis of clinical diabetes-are associated with higher likelihoods of prostate enlargement, $\mathrm{BPH}$, and LUTS. Higher serum concentrations of insulinlike growth factor-1 and insulin-like growth factor binding protein-3 have been associated with increased risk of clinical BPH and BPH surgery [34]. Physician-diagnosed diabetes, increased serum insulin, and elevated fasting plasma glucose have been associated with increased prostate size and increased risks of prostate enlargement, clinical BPH, BPH surgery, and LUTS in multiple different cohorts cumulatively incorporating tens of thousands of men $[30,31,34-36]$.

\section{Lipids}

Relatively few data are available on potential associations of lipids (high-density lipoprotein, low-density lipoprotein, and triglycerides) with BPH and LUTS. At least five studies have been conducted, with three showing positive and two showing null associations [31, 35, 37].

\section{Diet}

There are some indications that macronutrients and micronutrients may affect the risk of BPH and LUTS, 
although the patterns are somewhat inconsistent. For macronutrients, increased total energy intake, energyadjusted total protein intake, red meat, fat, milk and dairy products, cereals, bread, poultry, and starch potentially increase the risks of clinical BPH and BPH surgery; vegetables, fruits, polyunsaturated fatty acids, linoleic acid, and vitamin D potentially decrease the risks of BPH and LUTS [31, 38•]. With respect to micronutrients, higher circulating concentrations of vitamin E, lycopene, selenium, and carotene have been inversely associated with BPH and LUTS [31, 38•, 39]; zinc has been associated with increased and decreased risk [38• 39].

\section{Physical Activity}

Increased physical activity and exercise have been robustly and consistently linked with decreased risks of $\mathrm{BPH}$ surgery, clinical $\mathrm{BPH}$, histologic $\mathrm{BPH}$, and LUTS $[31,40]$. A meta-analysis of 11 published studies $(n=$ 43,083 men) indicated that moderate to vigorous physical activity reduced the risk of BPH or LUTS by as much as $25 \%$ relative to a sedentary lifestyle, with the magnitude of the protective effect increasing with higher levels of activity [41].

\section{Alcohol}

Like exercise, moderate alcohol intake also appears to be protective against multiple outcomes related to BPH [31]. However, unlike exercise, the same protective effect does not appear to apply to LUTS. A meta-analysis of 19 published studies ( $n=120,091 \mathrm{men})$ observed up to a $35 \%$ decreased likelihood of BPH among men who drank daily, but an increased risk of LUTS [42].

\section{Smoking}

Although several studies support the existence of an inverse protective effect of smoking on the risk of $\mathrm{BPH}$ and LUTS, several others have reported no risk or increased risk [31]. Thus, no definitive conclusions can be drawn at this time.

\section{Inflammation}

Most observational studies suggest that inflammation is intimately linked to the development of BPH and LUTS. The mechanisms underpinning this relationship are unclear. One potential explanation is that the metabolic syndrome, which promotes systemic inflammation and oxidative stress, mediates the connection between them [43]. Inflammation has been implicated as a primary stimulus for prostate carcinogenesis, and it is possible that
BPH represents a nonmalignant pathway of unregulated prostate growth promoted by oxidative stress, inflammatory mediators, and insulin growth pathways.

There are strong links between BPH and histologic inflammation in surgical specimens, with the extent and severity of the inflammation corresponding to the magnitude of prostate enlargement and BPH area [44-47]. Men with LUTS are more likely to have higher serum C-reactive protein, a robust marker of systemic inflammation [48], while prior gonorrheal infection or prostatitis increase the likelihood of BPH surgery and LUTS [49]. A history of infection with gonorrhea, chlamydia, or trichomonosis increases the risk of elevated prostate-specific antigen [50]; high serum $\operatorname{IgG}$ antibody titers to cytomegalovirus, herpes virus, human papilloma virus, and hepatitis are associated with LUTS [51].

Conversely, inhibition of inflammatory pathways potentially attenuates BPH risk. In one community cohort, men who reported daily NSAID use experienced significantly decreased risks of LUTS, low urinary flow rate, increased prostate volume, and elevated prostate-specific antigen [52].

\section{Conclusions}

In summary, BPH and LUTS are of significant importance to public health, affecting millions of older men and contributing to billions of dollars in health care cost each year. Current disease trends in the United States suggest that the number of men suffering from these conditions will swell markedly in the very near future. The rapid aging of the US population, coupled with the obesity and diabetes epidemics is poised to substantially increase the prevalence of BPH and LUTS within the general population and place even greater burdens on finite health care resources. Although the relatively immutable consequences of age and genetics factor substantially in the development of BPH and LUTS, many modifiable variables contribute as well-factors that may be manipulated to delay onset, prevent progression, or attenuate symptoms. Potential strategies include inhibition of DHT synthesis with $5-\alpha$ reductase inhibitors, modulation of metabolic risk factors with comprehensive lifestyle interventions incorporating diet change and physical activity, and suppression of inflammatory pathways with NSAIDs.

Acknowledgment Dr. Parsons has received grant funding from the National Institutes of Health.

Disclosure Dr. Parsons has served as a consultant for American Medical Systems and the University of California Physician Enhancement Program. 
Open Access This article is distributed under the terms of the Creative Commons Attribution Noncommercial License which permits any noncommercial use, distribution, and reproduction in any medium, provided the original author(s) and source are credited.

\section{References}

Papers of particular interest, published recently, have been highlighted as:

\section{- Of importance}

1. Wei JT, Calhoun E, Jacobsen SJ: Urologic diseases in America project: benign prostatic hyperplasia. J Urol 2005, 173:1256-1261.

2. Kupelian V, Wei JT, O'Leary MP, et al.: Prevalence of lower urinary tract symptoms and effect on quality of life in a racially and ethnically diverse random sample: the Boston Area Community Health (BACH) survey. Arch Intern Med 2006, 166:2381-2387.

3. Taylor BC, Wilt TJ, Fink HA, et al.: Prevalence, severity, and health correlates of lower urinary tract symptoms among older men: the MrOS study. Urology 2006, 68:804-809.

4. Parsons JK, Bergstrom J, Silberstein J, Barrett-Connor E: Prevalence and characteristics of lower urinary tract symptoms in men aged $>$ or $=80$ years. Urology 2008, 72:318-321.

5. Parsons JK, Wilt TJ, Wang PY, et al.; Osteoporotic Fractures in Men Research Group: Progression of lower urinary tract symptoms in older men: a community based study. J Urol 2010, 183:1915-1920.

6. Jacobsen SJ, Girman CJ, Guess HA, et al.: Natural history of prostatism: longitudinal changes in voiding symptoms in community dwelling men. J Urol 1996, 155:595-600.

7. Masumori N, Tsukamoto T, Rhodes T, Girman CJ: Natural history of lower urinary tract symptoms in men - result of a longitudinal community-based study in Japan. Urology 2003, 61:956-960.

8. Sarma AV, McLaughlin JC, Jacobsen SJ, et al.: Longitudinal changes in lower urinary tract symptoms among a cohort of black American men: the Flint Men's Health study. Urology 2004, 64:959-965.

9. Temml C, Brossner C, Schatzl G, et al.: The natural history of lower urinary tract symptoms over five years. Eur Urol 2003, 43:374-380.

10. Centers for Disease Control and Prevention: Trends in agingUnited States and worldwide. MMWR Morb Mortal Wkly Rep 2003, 52:101-104, 106.

11. Parsons JK, Mougey J, Lambert L, et al.: Lower urinary tract symptoms increase the risk of falls in older men. BJU Int 2009, 104:63-68.

12. Engstrom G, Henningsohn L, Walker-Engstrom ML, Leppert J: Impact on quality of life of different lower urinary tract symptoms in men measured by means of the SF 36 questionnaire. Scand J Urol Nephrol 2006, 40:485-494.

13. $\mathrm{Hu} \mathrm{TW}$, Wagner TH, Bentkover JD, et al.: Estimated economic costs of overactive bladder in the United States. Urology 2003, 61:1123-1128.

14. Saigal CS, Joyce G: Economic costs of benign prostatic hyperplasia in the private sector. J Urol 2005, 173:1309-1313.

15. Sanda MG, Beaty TH, Stutzman RE, et al.: Genetic susceptibility of benign prostatic hyperplasia. J Urol 1994, 152:115-119.

16. Pearson JD, Lei HH, Beaty $\mathrm{TH}$, et al.: Familial aggregation of bothersome benign prostatic hyperplasia symptoms. Urology 2003, 61:781-785.

17. Sanda MG, Doehring CB, Binkowitz B, et al.: Clinical and biological characteristics of familial benign prostatic hyperplasia. J Urol 1997, 157:876-879.
18. Rohrmann S, Fallin MD, Page WF, et al.: Concordance rates and modifiable risk factors for lower urinary tract symptoms in twins. Epidemiology 2006, 17:419-427.

19. Partin AW, Page WF, Lee BR, et al.: Concordance rates for benign prostatic disease among twins suggest hereditary influence. Urology 1994, 44:646-650.

20. Trifiro MD, Parsons JK, Palazzi-Churas K, et al.: Serum sex hormones and the 20-year risk of lower urinary tract symptoms in community-dwelling older men. BJU Int 2010, 105:1554-1559.

21. • Parsons JK, Palazzi-Churas K, Bergstrom J, Barrett-Connor E: A prospective study of serum dihydrotesterone and subsequent risk of benign prostatic hyperplasia in community dwelling men: the Rancho Bernardo study. J Urol 2010 Jul 17 (Epub ahead of print). This cohort study demonstrated that higher concentrations of serum DHT increase the risk of BPH. Serum total and bioavailable testosterone are not associated with BPH risk.

22. Kristal AR, Schenk JM, Song Y, et al.: Serum steroid and sex hormone-binding globulin concentrations and the risk of incident benign prostatic hyperplasia: results from the Prostate Cancer Prevention Trial. Am J Epidemiol 2008, 168:1416-1424.

23. Bhasin S, Singh AB, Mac RP, et al.: Managing the risks of prostate disease during testosterone replacement therapy in older men: recommendations for a standardized monitoring plan. J Androl 2003, 24:299-311.

24. Amory JK, Wang C, Swerdloff RS, et al.: The effect of 5alphareductase inhibition with dutasteride and finasteride on semen parameters and serum hormones in healthy men. J Clin Endocrinol Metab 2007, 92:1659-1665.

25. McConnell JD, Roehrborn CG, Bautista OM, et al.: The long-term effect of doxazosin, finasteride, and combination therapy on the clinical progression of benign prostatic hyperplasia. N Engl J Med 2003, 349:2387-2398.

26. Haffner S, Taegtmeyer H: Epidemic obesity and the metabolic syndrome. Circulation 2003, 108:1541-1545.

27. Meigs JB, Mohr B, Barry MJ, et al.: Risk factors for clinical benign prostatic hyperplasia in a community-based population of healthy aging men. J Clin Epidemiol 2001, 54:935-944.

28. Rohrmann S, Smit E, Giovannucci E, Platz EA: Association between markers of the metabolic syndrome and lower urinary tract symptoms in the Third National Health and Nutrition Examination Survey (NHANES III). Int J Obes (Lond) 2005, 29:310-316.

29. Joseph MA, Harlow SD, Wei JT, et al.: Risk factors for lower urinary tract symptoms in a population-based sample of AfricanAmerican men. Am J Epidemiol 2003, 157:906-914.

30. Parsons JK, Carter HB, Partin AW, et al.: Metabolic factors associated with benign prostatic hyperplasia. J Clin Endocrinol Metab 2006, 91:2562-2568.

31. Parsons JK: Modifiable risk factors for benign prostatic hyperplasia and lower urinary tract symptoms: new approaches to old problems. J Urol 2007, 178:395-401.

32. Parsons JK, Sarma AV, McVary K, Wei JT: Obesity and benign prostatic hyperplasia: clinical connections, emerging etiological paradigms and future directions. J Urol 2009, 182(6 Suppl):S27S31.

33. Kristal AR, Arnold KB, Schenk JM, et al.: Race/ethnicity, obesity, health related behaviors and the risk of symptomatic benign prostatic hyperplasia: results from the Prostate Cancer Prevention Trial. J Urol 2007, 177:1395-1400; quiz 591.

34. Sarma AV, Parsons JK, McVary K, Wei JT: Diabetes and benign prostatic hyperplasia/lower urinary tract symptoms - what do we know? J Urol 2009, 182(6 Suppl):S32-S37.

35. Parsons JK, Bergstrom J, Barrett-Connor E: Lipids, lipoproteins and the risk of benign prostatic hyperplasia in communitydwelling men. BJU Int 2008, 101:313-318.

36. Gupta A, Gupta S, Pavuk M, Roehrborn CG: Anthropometric and metabolic factors and risk of benign prostatic hyperplasia: a 
prospective cohort study of Air Force veterans. Urology 2006, 68:1198-1205.

37. Nandeesha H, Koner BC, Dorairajan LN, Sen SK: Hyperinsulinemia and dyslipidemia in non-diabetic benign prostatic hyperplasia. Clin Chim Acta 2006, 370:89-93.

38. - Kristal AR, Arnold KB, Schenk JM, et al.: Dietary patterns, supplement use, and the risk of symptomatic benign prostatic hyperplasia: results from the Prostate Cancer Prevention Trial. Am J Epidemiol 2008, 167:925-934. This analysis of the placebo arm of the Prostate Cancer Prevention Trial included several important observations with respect to diet and $B P H$, namely higher fat and red meat intake increase, while higher vegetable intake and regular alcohol consumption decrease BPH risk.

39. Tavani A, Longoni E, Bosetti $\mathrm{C}$, et al:: Intake of selected micronutrients and the risk of surgically treated benign prostatic hyperplasia: a case-control study from Italy. Eur Urol 2006, 50:549-554.

40. Dal Maso L, Zucchetto A, Tavani A, et al.: Lifetime occupational and recreational physical activity and risk of benign prostatic hyperplasia. Int J Cancer 2006, 118:2632-2635.

41. Parsons JK, Kashefi C: Physical activity, benign prostatic hyperplasia, and lower urinary tract symptoms. Eur Urol 2008, 53:1228-1235.

42. Parsons JK, Im R: Alcohol consumption is associated with a decreased risk of benign prostatic hyperplasia. J Urol 2009, 182:1463-1468.

43. Furukawa S, Fujita T, Shimabukuro M, et al.: Increased oxidative stress in obesity and its impact on metabolic syndrome. J Clin Invest 2004, 114:1752-1761.
44. Nickel JC, Downey J, Young I, Boag S: Asymptomatic inflammation and/or infection in benign prostatic hyperplasia. BJU Int 1999, 84:976-981.

45. Theyer G, Kramer G, Assmann I, et al.: Phenotypic characterization of infiltrating leukocytes in benign prostatic hyperplasia. Lab Invest 1992, 66:96-107.

46. Anim JT, Udo C, John B: Characterisation of inflammatory cells in benign prostatic hyperplasia. Acta Histochem 1998, 100:439-449.

47. Di Silverio F, Gentile V, De Matteis A, et al.: Distribution of inflammation, pre-malignant lesions, incidental carcinoma in histologically confirmed benign prostatic hyperplasia: a retrospective analysis. Eur Urol 2003, 43:164-175.

48. Rohrmann S, De Marzo AM, Smit E, et al.: Serum C-reactive protein concentration and lower urinary tract symptoms in older men in the Third National Health and Nutrition Examination Survey (NHANES III). Prostate 2005, 62:27-33.

49. Sutcliffe S, Giovannucci E, De Marzo AM, et al.: Sexually transmitted infections, prostatitis, ejaculation frequency, and the odds of lower urinary tract symptoms. Am J Epidemiol 2005, 162:898-906.

50. Sutcliffe S, Zenilman JM, Ghanem KG, et al.: Sexually transmitted infections and prostatic inflammation/cell damage as measured by serum prostate specific antigen concentration. J Urol 2006, 175:1937-1942.

51. Sutcliffe S, Rohrmann S, Giovannucci E, et al.: Viral infections and lower urinary tract symptoms in the Third National Health and Nutrition Examination Survey. J Urol 2007, 178:2181-2185.

52. St Sauver JL, Jacobson DJ, McGree ME, et al.: Protective association between nonsteroidal antiinflammatory drug use and measures of benign prostatic hyperplasia. Am J Epidemiol 2006, 164:760-768. 